This document is the accepted manuscript version of the following article:

Bello, C., \& Barreto, E. (2021). The footprint of evolution in seed dispersal interactions. The evolutionary stability of fruit-eating birds is linked to their role in dispersing seeds. Science, 372(6543), 682-683.

https://doi .org/10.1126/science. abi 8160

\title{
THE FOOTPRINT OF EVOLUTION IN SEED DISPERSAL INTERACTIONS
}

\author{
Carolina Bello $^{1 *}$, Elisa Barreto ${ }^{1,2}$
}

\begin{abstract}
${ }^{1}$ Swiss Federal Research Institute WSL. Zürcherstrasse 111. 8903 Birmensdorf, Switzerland. ${ }^{2}$ Laboratório de Ecologia Teórica e Síntese, Universidade Federal de Goiás (UFG), Goiânia, Goiás, Brazil

* Contact author: caro.bello58@gmail.com
\end{abstract}

Mutually beneficial interactions between plants and frugivorous birds have evolved for at least 80 million years (1). Currently, over $70 \%$ of flowering plants rely on birds to disperse their seeds, and about $56 \%$ of bird families consume fruits as part of their diet $(2,3)$. Plants often have more than one seed disperser, and birds consume different types of fruits from different plant species, thus, establishing a complex network of interactions. These networks of interactions can change considerably across space and time (4), and understanding the dynamics of it is a matter of great debate and relevance. Today, there is growing evidence that over short ecological time spans, seed-dispersal interactions are dynamic and change depending on factors such as competition or the presence and abundance of the interacting partner $(5,6)$. However, we still do not fully understand how evolutionary processes, taking place over deep time, influenced the current dynamics of seed dispersal interaction networks (7). Burin et al. shed light on the drivers of interactions by taking an evolutionary view of seed dispersal interaction networks. The authors detected the footprint of evolution by showing that evolutionary stability is associated with the species-specific role in seed dispersal networks.

The role of each species depends on the number of interactions it establishes with potential partner species and how this links to different modules of the network (8). So far, it has been known that modules that compounds these networks are a result from a series of ecological and evolutionary factors, such as co-evolution, trait convergence, and diversification $(9,10)$. Now, Burin adds one more piece to the puzzle by demonstrating that birds that play a central role in such a network (i.e., they interact with more plants and connect the modules of the network) tend to have greater persistence through evolutionary time. A lineage persists longer through time if it has lower extinction rates and/or higher speciation rates, which the authors named as "macroevolutionary stability". Having a low extinction rate implies longer longevities through evolutionary times, which in turn facilitate the establishment of more connections within the network as bird species have more time to co-evolve with plants. Whereas having high speciation rates suggest the accumulation of more sister species, who are expected to have similar traits and play similar role in the network, and therefore can act as a replacement if a species goes extinct (Figure 1). 


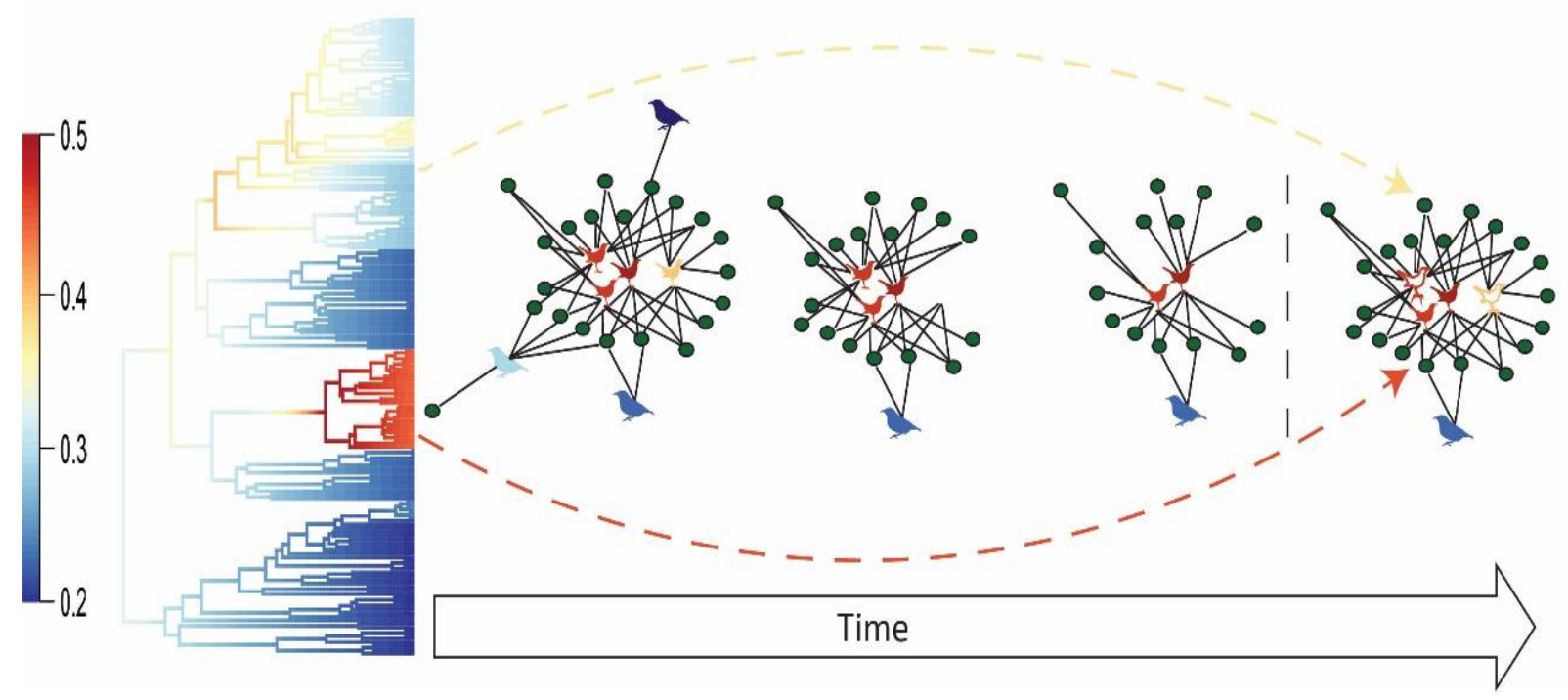

Figure 1. Graphical representation of the relationship between macroevolutionary stability and species' role in a seed dispersion interaction network. Birds and plants that interact are linked, forming a network of interactions that evolves through time. Plants are green circles, and birds' silhouettes are colored based on the evolutionary stability estimated from the phylogenetic tree. Red indicates greater stability, while blue indicates less stability. Birds that play a central role in networks (connect more plants and network modules) have greater evolutionary stability, that is, they persist for longer and/or generate more sister species. More stable species have a higher probability of being replaced by a sister species once they have gone extinct in the last network. Sister species are represented by the dashed lines and delineated silhouettes.

Understanding how evolutionary dynamics influence species interactions is an ongoing challenge that has persisted for so long, given the disparity in temporal scale between the observed networks and the macroevolutionary processes. Most studies on seed-dispersal assembly of networks are centered at ecological timescales and have shown that the cores of seed dispersal networks are stable to annual fluctuations in fruit availability or the presence of specific bird species (11). Studies that focused on evolutionary aspects of seed-dispersal networks were primarily centered on reconstructing trait evolution of the interacting species as a proxy to detect co-evolution (reviewed by Peralta (12). Besides, estimating speciation and, in particular extinction rates (13) accurately from phylogenies composed solely of extant species is still a challenge (e.g., Louca and Pennell (14)), making it hard to detect the footprint of evolutionary dynamics on species interactions. Although these methods are still controversial $(14,15)$, Burin et al. take the leap to merge macroevolution and interaction network and found a consistent and robust effect, even when accounting for the uncertainty of the rate estimates and the phylogenetic hypothesis.

Merging macroevolution and species interaction to understand the historical drivers of network assembly open a series of exciting questions to be explored in both evolutionary and ecological contexts. To begin, we still do not understand the direction and causality between macroevolutionary stability and the central role of a species in a network. Do bird species that persist over deep time become central in the interaction networks because they had more time to coevolve with the plants? Or are bird species that play a central role in interaction networks more resilient to temporal changes in the availability of resources, and therefore, have greater evolutionary stability? Or is it actually a combination of the two? Moreover, exploring the processes behind the relationship between macroevolution and species 
interactions will help us understand current ecological processes better. For example, is evolutionary stability related with a species' ability to become invasive, since invasive species generally interact with many species and occupy central positions in the networks? Which species traits are related to macroevolutionary stability and a species role in the network? Are interactions between species with different rates of speciation and extinction volatile under new climate scenarios? Exciting questions like these, which directly emerge from Burin's findings, open a new avenue for the field of interaction network research and can be of extreme importance to forecast the dynamics networks under new ecological conditions, especially given the current rates of climate change and ecosystem transformation.

Acknowledgements. We acknowledge funding support from the European Research Council (ERC) under the European Union's Horizon 2020 research and innovation program (grant agreement No 787638) and the Swiss National Science Foundation (grant No. 173342), both granted to Catherine Graham. We also thank Dirk Karger for his valuable comments to improve the manuscript.

\section{References}

1. O. Eriksson, Evolution of angiosperm seed disperser mutualisms: the timing of origins and their consequences for coevolutionary interactions between angiosperms and frugivores. Biol. Rev. Cambridge Philosophic. Soc. 91, 168-186 (2014).

2. T. H. Fleming, W. J. Kress, A brief history of fruits and frugivores. Acta Oecol.-Int. J. Ecol. 37, 521-530 (2011).

3. P. Jordano, Fruits and frugivory. Seeds: the ecology of regeneration in plant communities, 125-165 (2000).

4. P. J. CaraDonna et al., Seeing through the static: the temporal dimension of plant-animal mutualistic interactions. Ecology Letters 24, 149-161 (2021).

5. B. Rumeu, M. Álvarez-Villanueva, J. M. Arroyo, J. P. González-Varo, Interspecific competition for frugivores: population-level seed dispersal in contrasting fruiting communities. Oecologia 190, 605-617 (2019).

6. D. P. Vázquez et al., Species abundance and asymmetric interaction strength in ecological networks. Oikos 116, 1120-1127 (2007).

7. P. Jordano, Coevolution in multispecific interactions among free-living species. Evolution: Education and Outreach 3, 40-46 (2010).

8. P. Jordano et al., Frugivores and seed dispersal: mechanisms and consequences for biodiversity of a key ecological interaction. Biology Letters 7, 321-323 (2011).

9. C. I. Donatti et al., Analysis of a hyper-diverse seed dispersal network: modularity and underlying mechanisms. Ecology Letters 14, 773-781 (2011).

10. S. Chamberlain, D. P. Vázquez, L. Carvalheiro, E. Elle, J. C. Vamosi, Phylogenetic tree shape and the structure of mutualistic networks. Journal of Ecology 102, 1234-1243 (2014).

11. M. Plein et al., Constant properties of plant-frugivore networks despite fluctuations in fruit and bird communities in space and time. Ecology 94, 1296-1306 (2013).

12. G. Peralta, Merging evolutionary history into species interaction networks. Functional Ecology 30, 1917-1925 (2016).

13. D. L. Rabosky, Extinction rates should not be estimated from molecular phylogenies. Evolution: International Journal of Organic Evolution 64, 1816-1824 (2010).

14. S. Louca, M. W. Pennell, Extant timetrees are consistent with a myriad of diversification histories. Nature 580, 502-505 (2020).

15. B. R. Moore, S. Höhna, M. R. May, B. Rannala, J. P. Huelsenbeck, Critically evaluating the theory and performance of Bayesian analysis of macroevolutionary mixtures. Proceedings of the National Academy of Sciences 113, 9569-9574 (2016). 